\title{
Lu pour vous dans...
}

\section{La revue de Biologie médicale}

Par Laurence Piéroni

Bardet V. Les cytochimies hématologiques. Revue de Biologie Médicale 2020 ; 352 : 5.

Les colorations de Perls, des myéloperoxydases et des estérases restent très utiles, en particulier en complément du myélogramme standard coloré suivant la coloration de May-Grünwald-Giemsa. Elles sont toujours préconisées, selon l'Organisation mondiale de la santé (OMS), dans la distinction/classification des syndromes myélodysplasiques et des leucémies aiguës en dépit de l'importance accordée actuellement aux explorations par cytométrie en flux. Malgré leur longue histoire, les modalités de leur exécution restent hétérogènes : protocoles manuels locaux, peaufinés avec le temps, kits commerciaux avec leurs limites (manque de sensibilité parfois)... Comme pour la réalisation des myélogrammes, l'hétérogénéité des pratiques est grande. De plus, le processus d'accréditation EN-15189 des laboratoires entre désormais dans une phase avancée où les examens moins pratiqués sont en cours d'expertise, avec un besoin de textes d'harmonisation, de référentiels et de standards.

Lesesve JF, Andrieu V, Baccini V, Baseggio L, Brouzes C, Chatelain B, et al. pour le GFHC. Colorations de Perls, des myéloperoxydases, des estérases : enquête des pratiques et recommandations du groupe francophone d'hématologie cellulaire. Revue de Biologie Médicale 2020 ; 352 : 7-26.

Le Groupe francophone d'hématologie cellulaire (GFHC) est la société de référence pour tout sujet concernant la morphologie hématologique. Après la codification ADICAP des pathologies hématologiques, les recommandations concernant les valeurs de référence, l'hémogramme, le frottis sanguin et le myélogramme, le GFHC a souhaité recueillir l'expérience de ses membres sur la pratique des colorations cytochimiques. Soixante-sept centres volontaires ont répondu à un questionnaire et 101 morphologistes ont participé à l'enquête et/ou à l'élaboration des recommandations. L'exploration la plus courante est la coloration de Perls (100 \% des participants à l'enquête).
La détection des myéloperoxydases et des estérases est pratiquée par respectivement 70 et $30 \%$ des laboratoires ayant répondu à l'enquête. Un examen complémentaire cytochimique concerne environ un myélogramme sur 5 . Les réponses témoignent d'une homogénéité assez importante des pratiques (indications, analyse) et d'un accord sur les modalités d'interprétation et de rendu au prescripteur. Le GFHC présente ici des recommandations sur la détection cytochimique du fer insoluble, des myéloperoxydases et des estérases, avec pour objectif de répondre au mieux aux exigences de la classification OMS des hémopathies publiée en 2017. Le GFHC laisse à chaque laboratoire le choix des techniques à employer, protocoles locaux ou kits industriels. La conformité nécessaire avec la norme EN-15189 sera néanmoins facilitée par l'usage de kits de détection respectant la nouvelle réglementation européenne sur les dispositifs médicaux de diagnostic in vitro. En raison de la toxicité de certains composants, une grande vigilance est recommandée sur le respect des consignes d'utilisation et de sécurité pour le personnel utilisateur.

Geneviève F, Lesesve JF, Andrieu V, Girard S, Chatelain B. Coloration de Perls : un exemple de mode opératoire. Revue de Biologie Médicale 2020 ; 352 : 27-29.

La coloration de Perls a pour but de mettre en évidence le fer insoluble précipité dans le cytoplasme des érythroblastes et du macrophage. Les modalités de sa réalisation sont décrites selon un protocole non commercialisé, dans le but de fournir à d'éventuels utilisateurs un mode opératoire indépendant des fabricants de réactifs de laboratoire

Rault E, Fenneteau O, Bardet V, Lesesve JF. Présence de sidéroblastes en couronne en dehors du contexte de syndrome myélodysplasique : que faut-il évoquer? Revue de Biologie Médicale 2020 ; 352 : 31-6.

Bien que l'excès de sidéroblastes en couronne soit un critère essentiel de certains sous-types de syndromes myélodysplasiques (SMD), cette anomalie cytologique n'est pas pour autant spécifique des SMD. Nous rappelons ici les principales circonstances dans lesquelles une sidéroblastose 
peut être observée. Les exceptionnelles anémies sidéroblastiques congénitales et les cytopathies mitochondriales surviennent dans des contextes cliniques particuliers. Les causes acquises sont les plus fréquentes chez l'adulte et liées à une exposition à des produits toxiques ou à la prise de certains médicaments.

Freynet N, Geneviève F, Lesesve JF, Wagner-Ballon O. Mise en évidence des myéloperoxydases : un exemple de mode opératoire. Revue de Biologie Médicale 2020 ; 352 : 37-40.

Les modalités de réalisation des colorations cytochimiques permettant de mettre en évidence la myéloperoxydase (MPO) sont décrites avec un protocole sensible et non commercialisé. La liste des kits industriels est proposée à titre indicatif. La détection de la MPO dans le cytoplasme des cellules permet de déterminer leur nature myéloïde, en particulier pour le classement initial d'une leucémie aiguë.

Girard S, Lesesve JF, Chatelain B. Mise en évidence des estérases : un exemple de mode opératoire. Revue de Biologie Médicale 2020 ; 352 : 41-46.

Les réactions cytochimiques détectant l'activité estérasique sur des frottis de moelle osseuse et/ou de sang sont principalement effectuées lors du bilan diagnostique d'une leucémie aiguë, en complément de l'analyse cellulaire après coloration de May-Grünwald-Giemsa. Elles permettent d'affirmer la nature monocytaire des blastes. Deux protocoles (estérases spécifiques et non spécifiques) sont commentés.

Peoc'h K, Castera L. Marqueurs non invasifs de stéatopathie métabolique. Revue de Biologie Médicale 2020 ; 352 : 47-52.

La stéatopathie métabolique (NAFLD : nonalcoholic Jatty liver disease) affecte environ un quart de la population générale et représente désormais la principale cause de maladie chronique du foie en Occident. La stéatohépatite non alcoolique ou NASH, qui constitue la forme active de la NAFLD, est définie par l'association à la stéatose, de lésions de ballonisation hépatocytaire et d'inflammation lobulaire. Les deux enjeux diagnostiques majeurs chez les patients atteints de stéatopathie métabolique sont la différenciation entre une NASH et une stéatose isolée bénigne et l'identification d'une fibrose hépatique sévère ou d'une cirrhose (stades F3-F4). Les avantages des biomarqueurs sanguins comprennent leur applicabilité élevée (> $95 \%$ ), leur bonne reproductibilité inter-laboratoire et leur large disponibilité. En revanche, aucun marqueur n'est vraiment spécifique de la NASH ou de la fibrose. Concernant l'identification des patients avec une fibrose hépatique sévère ou une cirrhose, les scores les mieux validés sont le FIB-4 et le NFS (NAFLD fibrosis score), avec des AUROC (area under receiver opeating characteristic) aux alentours 0,82 et des valeurs prédictives négatives supérieures à $90 \%$.

Sawant M, Benamrouz S, Chabé M, Guyot K, Costa D, Favennec L, et al. La cryptosporidiose et son impact en santé publique. Revue de Biologie Médicale 2020 ; 352 : 53-68.

Cryptosporidium est une cause majeure de diarrhée à travers le monde et l'infection par ce parasite touche en particulier les jeunes enfants et les patients immunodéprimés. La gravité de l'infection intestinale induite par ce protozoaire intracellulaire est variable et les symptômes cliniques de la cryptosporidiose dépendent non seulement de l'état nutritionnel et immunitaire de l'hôte mais également des facteurs de virulence du parasite. L'infection humaine résulte d'une propagation zoonotique (de l'animal à l'homme) ou anthroponotique (inter-humaine) du parasite dont la transmission est facilitée par sa résistance aux nombreux désinfectants couramment utilisés. Les mesures de prévention et de contrôle de l'infection sont capitales pour la protection des groupes vulnérables car les options de traitement restent encore limitées.

Ourabah R. Qu'attend le médecin généraliste de la prescription des examens biologiques ? Revue de Biologie Médicale 2020 ; 352 : 69-72.

Les examens biologiques sont largement prescrits par le médecin généraliste, classiquement dans le but d'établir un diagnostic ou de surveiller une pathologie connue. La prise en charge d'un patient en médecine générale est rarement ponctuelle ; elle s'étend le plus souvent sur une longue période de sa vie. Ainsi, depuis le moment précédant l'apparition d'une maladie, son diagnostic, sa prise en charge et son suivi, la prescription des examens biologiques doit répondre à chacun de ces moments et à chacune de ces circonstances. Le prescripteur attend pour chacun d'eux une réponse du biologiste adaptée à la circonstance de la prescription 